\title{
Telomeric heterochromatin boundaries require NuA4-dependent acetylation of histone variant H2A.Z in Saccharomyces cerevisiae
}

\author{
Joshua E. Babiarz, Jeffrey E. Halley, and Jasper Rine ${ }^{1}$ \\ Department of Molecular and Cell Biology, University of California at Berkeley, Berkeley, California 94720-3202, USA
}

\begin{abstract}
SWR1-Com, which is responsible for depositing H2A.Z into chromatin, shares four subunits with the NuA4 histone acetyltransferase complex. This overlap in composition led us to test whether H2A.Z was a substrate of NuA4 in vitro and in vivo. The $\mathrm{N}$-terminal tail of H2A.Z was acetylated in vivo at multiple sites by a combination of NuA4 and SAGA. H2A.Z acetylation was also dependent on SWR1-Com, causing H2A.Z to be efficiently acetylated only when incorporated in chromatin. Unacetylatable H2A.Z mutants were, like wild-type H2A.Z, enriched at heterochromatin boundaries, but were unable to block spreading of heterochromatin. A mutant version of $\mathrm{H} 2 \mathrm{~A} . \mathrm{Z}$ that could not be acetylated, in combination with a mutation in a nonessential gene in the NuA4 complex, caused a pronounced decrease in growth rate. This H2A.Z mutation was lethal in combination with a mutant version of histone $\mathrm{H} 4$ that could not be acetylated by NuA4. Taken together, these results show a role for H2A.Z acetylation in restricting silent chromatin, and reveal that acetylation of $\mathrm{H} 2 \mathrm{~A} . \mathrm{Z}$ and $\mathrm{H} 4$ can contribute to a common function essential to life.
\end{abstract}

[Keywords: Silencing; SIR proteins; histone code; HTZ1]

Supplemental material is available at http://www.genesdev.org.

Received October 19, 2005; revised version accepted January 20, 2006.

Differences in the pattern of histone modifications found at different places in the genome lead to unique properties of the chromatin at these positions. These links have been formalized as the histone code hypothesis (Strahl and Allis 2000; Peterson and Laniel 2004). Histone variants substitute for canonical histones in certain highly specialized chromatin structures, but unlike canonical histones, modification of histone variants is largely unexplored. Not all modifications can be considered unique components of a code. For instance, acetylation of Lys 5, Lsy 8, and Lys 12 of histone $\mathrm{H} 4$ contribute quantitatively to gene expression across the yeast genome. This is in contrast to acetylation of Lys 16 of histone H4, which has distinct consequences (Dion et al. 2005). Histone H4 Lys 16 acetylation is a hallmark of euchromatin, and hypoacetylation of this position is a hallmark of heterochromatin. H4 Lys 16 is acetylated by the SAS histone acetyltransferase (HAT) complex (Kimura et al. 2002; Suka et al. 2002). Heterochromatin is found in three locations in the Saccharomyces cerevisiae genome: the cryptic mating loci (HML and $H M R)$, telomeres and

${ }^{1}$ Corresponding author.

E-MAIL jrine@berkeley.edu; FAX (510) 642-6420.

Article and publication are at http://www.genesdev.org/cgi/doi/10.1101/ gad.1386306. some subtelomeric regions, and ribosomal DNA (Rusche et al. 2003). Silencing at the cryptic mating loci and telomeres is mediated by the Silent-Information Regulator $(S I R)$ proteins, and is caused in part by histone deacetylation. Sir2 protein is a histone deacetylase whose preferred substrates include H4 Lys 16. Sir3p and Sir4p are histone tail-binding proteins, and Sir3p preferentially binds unmodified histone $\mathrm{H} 4$ tails in vitro and presumably in vivo as well (Carmen et al. 2002).

Post-translational acetylation and deacetylation of histones are carried out by large, multisubunit complexes. In $S$. cerevisiae, NuA4 is the only essential HAT complex (Smith et al. 1998; Clarke et al. 1999). Deletion of the catalytic subunit of NuA4, encoded by ESA1, results in inviability. Temperature-sensitive alleles of ESA1 cause reduced acetylation of histones $\mathrm{H} 2 \mathrm{~A}$ and $\mathrm{H} 4$, and sensitivity to genotoxic stress by drugs such as methyl methanesulfonate and camptothecin (Bird et al. 2002). In addition, ESA1 has a role in DNA double-strand break repair. The SAGA complex is another HAT that acetylates histone $\mathrm{H} 3$ through its catalytic subunit encoded by GCN5 (Trievel et al. 1999; Timmers and Tora 2005).

The dynamic structure of chromatin involves more than just reversible covalent modifications. The replacement of canonical histones with minor histone variants at particular positions in the genome also has specific 
consequences (Kamakaka and Biggins 2005; Sarma and Reinberg 2005). For example, CENP-A (Cse4p in S. cerevisiae) is a variant of $\mathrm{H} 3$ present at eukaryotic centromeres that is essential for viability, presumably due to its role in centromere function. However, modifications of histone variants are just beginning to be explored and, in the few cases studied, appear to be important in their function. H2A.X is a histone H2A variant that is randomly deposited but is phosphorylated at the site of double-stranded DNA breaks. This phosphorylation is an early response to double-strand DNA breaks and is important for maintaining the repair machinery at the site of the break.

The CSE4-encoded H3 variant and the HTZ1-encoded $\mathrm{H} 2 \mathrm{~A}$. Z are the only yeast histone variants. H2A.Z is conserved from yeast to humans and is essential for viability in all organisms tested except Saccharomyces and Schizosaccharomyces pombe (Redon et al. 2002). Hence yeast offers an optimum context to unravel the functions of H2A.Z and its modifications. Yeast cells lacking H2A.Z are sensitive to stress by chemicals such as hydroxyurea, caffeine, formamide, and benomyl (Kobor et al. 2004). In addition, H2A.Z helps determine the boundary of silent heterochromatin. A deletion of HTZ1 results in the spreading of the Sir proteins from heterochromatin into euchromatin and a reduction in transcription of genes adjacent to silent domains (Meneghini et al. 2003).

A major breakthrough in understanding H2A.Z came from the identification of the multisubunit protein complex known as SWR1-Com, which deposits H2A.Z into chromatin (Krogan et al. 2003; Kobor et al. 2004; Mizuguchi et al. 2004). The SWR1-Com, named by convention after its AAA-ATPase subunit, shares four subunits with the NuA4 HAT complex, and shares an overlapping set of four subunits with the Ino80 chromatin remodeling complex (Kobor et al. 2004; Krogan et al. 2004). Furthermore, the human NuA4 complex contains the SWR1 homolog p400/hDomino, establishing the generality of subunit sharing between Swr1-com and NuA4, although the specific subunits that are shared vary from species to species (Doyon and Cote 2004; Doyon et al. 2004).

In Tetrahymena, H2A.Z is multiply acetylated on a repeated GGK motif and on one GGAK motif by an unknown HAT(s) (Ren and Gorovsky 2001, 2003). However, this motif is present only once in S. cerevisiae's H2A.Z. In addition, H2A.Z acetylation is essential for viability in Tetrahymena, complicating functional analysis (Ren and Gorovsky 2001). In vitro studies establish that the Drosophila $\mathrm{H} 2 \mathrm{~A}$ variant $\mathrm{H} 2 \mathrm{Av}$, which shares properties with H2A.Z and H2A.X, can be acetylated by the Drosophila Tip60 complex, the analog of yeast NuA4 (Kusch et al. 2004). This acetylation is a prerequisite for removal of phosphorylated $\mathrm{H} 2 \mathrm{Av}$ at the site of DNA double-stranded breaks. However Drosophila $\mathrm{H} 2 \mathrm{Av}$ is a hybrid of H2A.Z and H2A.X sequences, which complicates extrapolating the function of H2A.Z acetylation to other species.

The present study investigated the acetylation state of S. cerevisiae H2A.Z in vivo, the enzyme(s) responsible, and the role(s) of these modifications. The subunits shared by SWR1-Com and NuA4 led us to test whether H2A.Z, a substrate for SWR1-Com, was also a substrate for NuA4. As described below, the answer to this question revealed unanticipated complexity in the modifications of H2A.Z, and established a surprising essential role for $\mathrm{H} 2 \mathrm{~A} . \mathrm{Z}$ acetylation, even though $\mathrm{H} 2 \mathrm{~A} . \mathrm{Z}$ itself is not essential.

\section{Results}

H2A.Z was a substrate for NuA4 in vitro

The SWR1-Com deposits H2A.Z into chromatin and shares several subunits with the NuA4 HAT complex (Krogan et al. 2003; Kobor et al. 2004; Mizuguchi et al. 2004). This sharing of subunits suggested that H2A.Z may be a substrate for NuA4. To test this hypothesis, $S$. cerevisiae H2A.Z was tested for whether it could be acetylated by NuA4. In these experiments, H2A.Z was expressed in Escherichia coli and purified from inclusion bodies. H2A.Z was then reconstituted into dimers with $\mathrm{H} 2 \mathrm{~B}$, and into octamers with $S$. cerevisiae histones H2B, $\mathrm{H} 3$, and $\mathrm{H} 4$ that had also been purified following expression in E. coli. Hence these histones lacked eukaryotic modifications. Native NuA4 complex was purified from $S$. cerevisiae using a strain that carried a TAP-tagged version of Esalp, the catalytic subunit of NuA4.

Following purification, the various histone complexes were incubated with purified NuA4 in the presence of ${ }^{3} \mathrm{H}$ Acetyl-CoA. NuA4 efficiently acetylated H4 and H2A. The strong signal from the position in the gel corresponding to H2A.Z either as H2A.Z-H2B dimers or as octamers defined H2A.Z as a new substrate of the NuA4 complex in vitro (Fig. 1A). However, under the salt concentrations of these assays, the octamers fall apart into

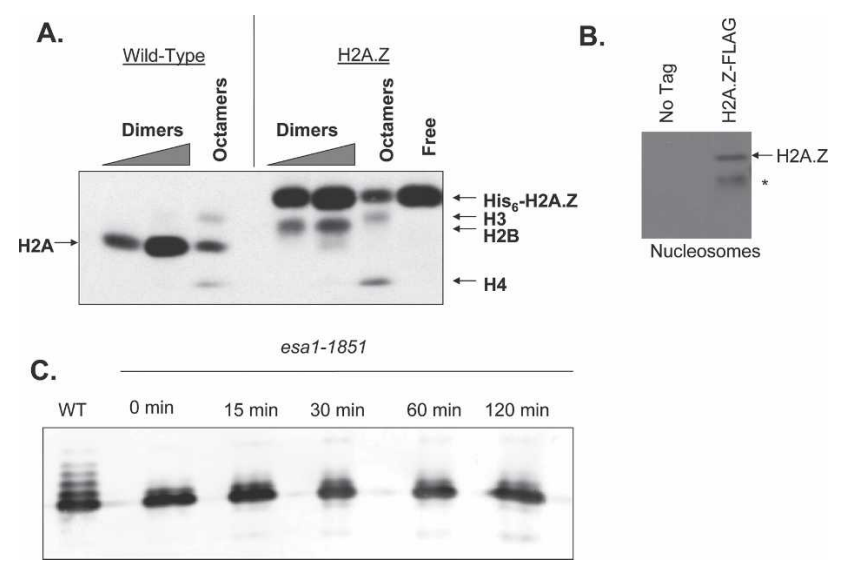

Figure 1. H2A.Z was acetylated by NuA4 in vitro and in vivo. (A) ${ }^{3} \mathrm{H}$-Acetyl-CoA was incorporated into H2A and H2A.Z by NuA4. (B) ${ }^{3} \mathrm{H}$-Acetyl-CoA was incorporated into H2A.Z-containing nucleosomes by NuA4. (C) H2A.Z was acetylated in vivo primarily by NuA4. The image represents an anti-Flag immunoblotting of an $\mathrm{AU}$ gel with times referring to minutes past shift to $37^{\circ} \mathrm{C}$. 
H3-H4 tetramers and H2A.Z-H2B dimers. To determine if NuA4 could acetylate H2A.Z-containing nucleosomes, chromatin was purified from a strain that was deficient for NuA4 (eaf1 $\Delta$ ) and that contained a C-terminal triple-Flag-tagged version of H2A.Z expressed from the HTZ1 locus. This tagged version of H2A.Z complemented all htz1s phenotypes (data not shown). Nucleosomes were released by micrococcal nuclease (MNase) treatment, and H2A.Z nucleosomes were purified using $\alpha$ Flag affinity resin. Native NuA4 complex was added along with ${ }^{3} \mathrm{H}$ Acetyl-CoA. H2A.Z was acetylated when present in nucleosomes (Fig. 1B). As observed for Drosophila $\mathrm{H} 2 \mathrm{Av}$, NuA4 preferentially acetylated H2A.Z over $\mathrm{H} 4$ contained in the same nucleosome (Kusch et al. 2004). In addition to H2A.Z, a second protein was acetylated whose mobility is consistent with a protein the size of either $\mathrm{H} 2 \mathrm{~A}$ or $\mathrm{H} 2 \mathrm{~B}$. One possibility is $\mathrm{H} 2 \mathrm{~B}$, as NuA4 acetylation of $\mathrm{H} 2 \mathrm{~B}$ is observed in $\mathrm{H} 2 \mathrm{~A} . \mathrm{Z}-\mathrm{H} 2 \mathrm{~B}$ dimers (Fig. 1A). A second possibility is H2A. It has been shown recently that $\mathrm{H} 2 \mathrm{~A} . \mathrm{Z}$ can form mixed nucleosomes with H2A in vitro (Chakravarthy et al. 2004). H2A.Z-containing nucleosomes purified using this method contain H2A, presumably in nucleosomes carrying one $\mathrm{H} 2 \mathrm{~A}$ molecule and one H2A.Z (data not shown).

In vivo, NuA4 acetylates three lysines on histone $\mathrm{H} 4$ and two on H2A (Suka et al. 2001). To determine the number of acetyl groups that were added to H2A.Z by NuA4 in vitro, we used ion-trap mass spectrometry. The in vitro reaction was performed as described above using free H2A.Z as a substrate. Mass spectrometry analysis identified a peak at the predicted molecular weight of H2A.Z and, only in the presence of acetyl-CoA, a peak corresponding to H2A.Z + $42 \mathrm{Da}$, the molecular weight of a single acetyl group. No additional peaks suggesting di- or triacetylation were observed (data not shown). These data established that H2A.Z could be a direct substrate for NuA4. However, the in vivo experiments (below), revealed multiple sites of acetylation.

\section{H2A.Z was a substrate for NuA4 in vivo}

To determine whether H2A.Z was acetylated by NuA4 in vivo, we used acetic acid-urea gels (AU gels) to separate histone isoforms based on size and charge (Lennox and Cohen 1989). Since acetylation of a lysine neutralizes its positive charge, acetylated histones migrate more slowly then unmodified versions, with single-acetylation resolution possible. Because $\mathrm{H} 2 \mathrm{~A}$. $\mathrm{Z}$ is a minor histone variant, with an abundance $\sim 10 \%$ that of $\mathrm{H} 2 \mathrm{~A}$, we first immunoprecipitated H2A.Z-3Flag such that the amounts of H2A.Z exceeded the binding capacity of the $\alpha$ Flag affinity resin used. This was done so that minor differences in absolute H2A.Z amounts between different mutant strains would not influence our interpretations. The immunoprecipitated H2A.Z was separated by AU gels, transferred to a membrane, subject to $\alpha$ Flag immunoblots, so that the signal from H2A.Z would not be masked by that from H2A (Ren and Gorovsky 2001, 2003).
On AU gels, H2A.Z had at least four modified forms that migrated more slowly than the fastest migrating and presumptively unmodified band, suggesting multiple acetylations, or phosphorylations, or both (Fig. 1C). This result contrasted with the in vitro data suggesting that H2A.Z was monoacetylated by NuA4. The difference in results from the two assays may reflect differences between in vivo and in vitro HAT specificities, or modification of H2A.Z by multiple HATs in vivo.

To test whether one or more of these modified forms of H2A.Z was due to the function of the NuA4 HAT, we expressed H2A.Z-3Flag in a strain with a severe temperature-sensitive allele of ESA1, the gene encoding the catalytic subunit of NuA4. esa1-1851 strains have defects in histone $\mathrm{H} 4$ acetylation even at the permissive temperature (Bird et al. 2002). If H2A.Z was acetylated in vivo by NuA4, then the esa1-1851 allele would cause a reduction in the amount of slowly migrating, and therefore acetylated, forms of H2A.Z (Fig. 1C). Three of the four modified forms of H2A.Z were eliminated at both the permissive and restrictive temperature, leaving a single NuA4independent modification. These data identified the NuA4 HAT as being responsible for $\mathrm{H} 2 \mathrm{~A}$. Z modification in vivo. The in vitro data above suggested that at least one of these forms resulted from direct acetylation of H2A.Z by NuA4. Whether NuA4 was responsible for the other species, or if other HATs add those modifications in a NuA4-dependent manner, is resolved below.

To determine if the reduction in the number of modified species of H2A.Z was specific to the Esalp subunit of NuA4, we evaluated H2A.Z modification in an eaf1s mutant. EAF1 encodes a nonessential subunit of NuA4, and eaf1s mutants, although viable, have histone $\mathrm{H} 4$ acetylation defects (Kobor et al. 2004). H2A.Z-3Flag in an eaf1 $\Delta$ mutant produced a similar modification pattern to esa1-1851 (Fig. 2A) on AU gels. Therefore, two different
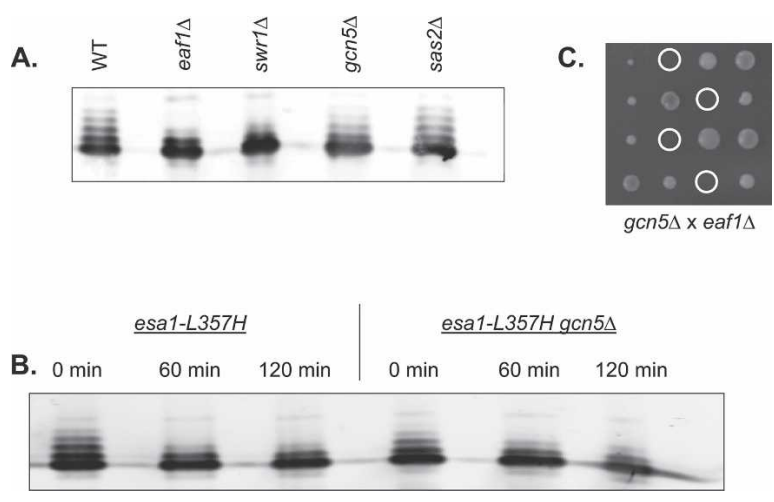

Figure 2. Other enzymes contributed to H2A.Z acetylation. $(A-C)$ Shown are anti-Flag immunoblots of an AU gel. (A) Several genes encoding the catalytic subunits of HATs and the SWR1-Com were deleted and analyzed for defects in H2A.Z acetylation. (B) Epistasis analysis of esa1-L357H and gcn5s showed that NuA4 acetylated H2A.Z prior to SAGA acetylation. $(C) g c n 5 \Delta$ and eaf $1 \Delta$ were synthetic lethal as shown by four tetra-type tetrads. The white circles represent where double mutants should be observed. 
disruptions of the NuA4 complex had similar phenotypes with respect to $\mathrm{H} 2 \mathrm{~A} . \mathrm{Z}$ modifications.

\section{SAGA acetylated H2A.Z in vivo}

To determine the source of the remaining modifications of H2A.Z in a cell compromised for NuA4 activity, we tested the contribution of other HATs to H2A.Z acetylation. GCN5 and SAS2 were of particular interest, as gcn5s htz1s double mutants exhibit a striking slowgrowth phenotype (Santisteban et al. 2000), and SAS2, like HTZ1, helps to constrain the spread of heterochromatin (Kimura et al. 2002; Suka et al. 2002). GCN5 encodes the catalytic subunit of SAGA. gcn5 $\Delta$ mutant cells had a modest yet clear reduction of the number of modified forms of H2A.Z (Fig. 2A). Instead of the four modified forms of H2A.Z in wild type, there were two modified forms, with a third modified form of low abundance in a gcn $5 \Delta$ mutant. These data suggested that SAGA added a single acetyl group. In contrast, loss of Sas $2 p$ had no effect on H2A.Z modifications (Fig. 2A).

Given that both SAGA and NuA4 contributed to H2A.Z acetylation in vivo, we tested whether the residual modification of H2A.Z in an esa1-1851 and eaf1s mutant was due to acetylation by SAGA. Unexpectedly, the $g c n 5 \Delta$ and eaf1s (SAGA and NuA4) double-mutant combination was lethal (Fig. 2C). Because gcn5s would also likely be lethal in combination with the severe esa11851 allele, we introduced the gcn5 $\Delta$ allele into a strain carrying the milder esa1-L357H mutation. esa1-L357H strains exhibited normal histone $\mathrm{H} 4$ acetylation at the permissive temperature (Bird et al. 2002), as well as a normal H2A.Z-3Flag acetylation pattern at the permissive temperature (Fig. 2B). Upon shifting esa1-L357H to restrictive temperature for 60 or $120 \mathrm{~min}$, the acetylation pattern looked similar to esa1-1851 and eaf1s. The esa1$\mathrm{L} 357 \mathrm{H}$ allele was viable in combination with gcn5 ; however, these cells grew notably more slowly than either single mutant (data not shown). At permissive temperature, the esa1- $2357 \mathrm{H} \mathrm{gcn} 5 \Delta \mathrm{H} 2 \mathrm{~A} . \mathrm{Z}$ acetylation pattern appeared to be similar to gcn5s (Fig. 2, cf. A and B). Shifting these cells to nonpermissive temperature did not result in the loss of the residual H2A.Z modification. Thus, the remaining modification was not due to SAGA. This epistasis analysis suggested that NuA4 acetylation of H2A.Z was a prerequisite for SAGA acetylation.

\section{SWR1-Com was necessary for H2A.Z acetylation}

The sharing of subunits between SWR1-Com and NuA4 suggested two different models for the roles of these two complexes in the incorporation of H2A.Z into chromatin. In one model, SWR1-Com would deposit H2A.Z into chromatin, and the shared subunits with NuA4 would then recruit that complex to acetylate H2A.Z. In the alternative model, H2A.Z would require prior acetylation by NuA4 to either interact with SWR1-Com or to be incorporated into chromatin by SWR1-Com. H2A.Z in a swr1s mutant showed reduction in H2A.Z acetylation similar to reduction exhibited in the NuA4 mutant strains (Fig. 2A). Thus acetylation of H2A.Z by NuA4 appeared to require prior incorporation into chromatin by SWR1-Com.

To provide an independent test of the dependency relationships between chromatin association and H2A.Z acetylation, we evaluated the modifications present on both the chromatin-associated and soluble fractions of H2A.Z. Spheroplasted cells were gently lysed with a nondenaturing detergent, and the chromatin was separated from the soluble non-chromatin-bound proteins. The chromatin was subsequently digested with micrococcal nuclease to release individual nucleosomes (Donovan et al. 1997; Liang and Stillman 1997). As expected from analysis of the swr1s mutant (above), soluble H2A.Z was largely unacetylated (Fig. 3), and the soluble fraction contains the majority of the unmodified H2A.Z in the cell. In swr1s cells, soluble H2A.Z was similarly unmodified, but the chromatin-associated fraction had significantly reduced acetylation compared with wild type (Fig. 3). Thus, efficient acetylation of H2A.Z by NuA4 could not occur in the absence of SWR1-Com.

\section{H2A. $Z$ was acetylated in the $N$-terminal tail}

The four lysines in the N-terminal tail of H2A.Z at positions $3,8,10$, and 14 provided a potential explanation for the four modified forms of H2A.Z evident in the AU gels. These positions in HTZ1-3Flag were mutated to arginine codons, to mimic the unacetylated state, creating htz1-K3,8,10,14R-3Flag. This mutant H2A.Z was largely unmodified, as judged by mobility on AU gels (Fig. 4A). Comparing the H2A.Z mobility in an eaf1 $\Delta$ and $h t z 1-K 3,8,10,14 R$ (Fig. 4A) revealed that H2A.Z was more severely unmodified in the $h t z 1-K 3,8,10,14 R$ mutant than in the eaf1 $\Delta$ mutant. The remaining modified species in the $h t z 1-K 3,8,10,14 R$ mutant was in low abundance, and might reflect acetylation at a site other than the N-terminal tail, or perhaps phosphorylation elsewhere in the protein. As expected, this mutant could not be acetylated by NuA4 in vitro (data not shown).

To determine the acetylated sites of H2A.Z, the modification state was monitored in cells with individual $\mathrm{N}$ terminal lysines changed to arginine (Fig. 4B). All four individual lysine mutants appeared similar, exhibiting loss of a modified form on AU gels, suggesting that no one residue was more important for acetylation than the

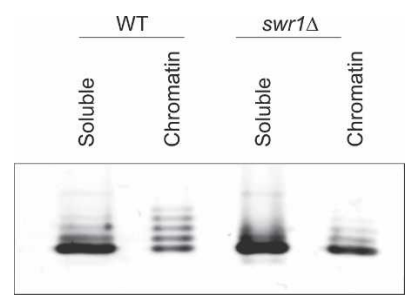

Figure 3. Soluble and chromatin-associated H2A.Z was analyzed for acetylation in wild-type and swr1s. Shown is an antiFlag immunoblot of an AU gel. 

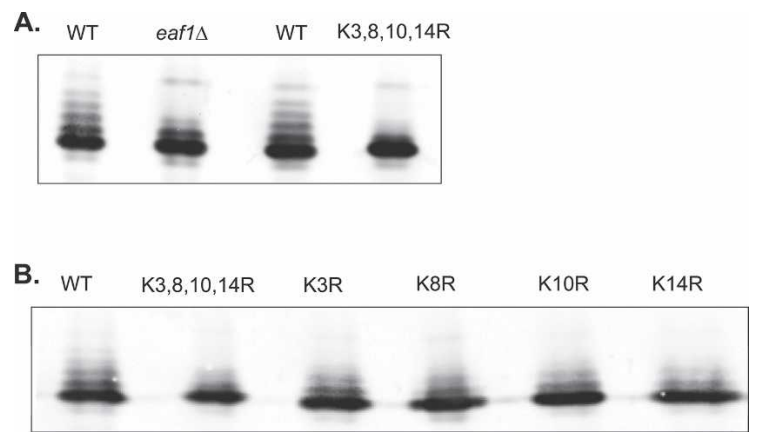

Figure 4. H2A.Z was acetylated in the N-terminal tail. $(A, B)$ Shown are anti-Flag immunoblots of an AU gel. (A) H2A.Z is acetylated at the N-terminal Lys 3, Lys 8, Lys 10, and Lys 14. Lanes 1 and 2 are chromosomal HTZ1-3Flag. Lanes 3 and 4 are plasmid-borne HTZ1-3Flag alleles. (B) Lys 3, Lys 8, Lys 10, and Lys 14 can all be acetylated in vivo. All HTZ1-3Flag alleles are plasmid-borne.

other. In addition, loss of one modified form of H2A.Z in each mutant suggests that each site is subject to acetylation. (Fig. 4B)

\section{H2A.Z acetylation blocked the spread of silencing}

The boundaries that restrict the spread of heterochromatin are compromised in $h t z 1 \Delta$ mutants (Meneghini et al. 2003). To test whether H2A.Z acetylation was important for the function of these boundaries, quantitative RTPCR (qRT-PCR) was used to quantitate mRNA levels of genes potentially susceptible to heterochromatic position effects in wild-type, $h t z 1 \Delta$, and $h t z 1-K 3,8,10,14 R$ strains. The expression of four genes was monitored: YPS6 and YIR042c are near the telomeres of chromosome IX-R; YCR095c and GIT1 are near the HMR left and right boundaries, respectively. ACT1, whose mRNA level does not change in an htz1s mutant (Meneghini et al. 2003), was used for normalization. The unacetylatable $h t z 1-K 3,8,10,14 R$ mutant was defective in boundary function, as reflected by the reduction in the expression level of YPS6 and YIR042c in the unacetylatable mutant compared with wild type (Fig. 5A). This repression was entirely Sir protein-dependent, as a deletion of SIR2 in the $h t z 1-K 3,8,10,14 R$ mutant restored wild-type expression levels. In principal, these results could have been due to a mislocalization of H2A.Z, because the silencing defect in $h t z 1-K 3,8,10,14 R$ cells was similar to that of htz1s cells. However, chromatin immunoprecipitation (ChIP) of HTZ1-3Flag and htz1-K3,8,10,14R-3Flag showed similar enrichment at YPS6 and YIR042c compared with the negative control, PRP8 (Fig. 5C), establishing that the loss of boundary function was due to the loss of H2A.Z acetylation and not due to mislocalization of H2A.Z. As an independent test of the boundary function of the mutant H2A.Z, we tested whether the SIR proteins were spread inward from the telomeres beyond their position in wild-type cells, as would be predicted from the reduced expression of subtelomeric genes. In this experiment, SIR3 was TAP-tagged in both HTZ1 and htz1-K3,8,10,14R strains. ChIP of Sir3-TAP followed by qPCR showed a gradient of Sir3p at telomere IX-R, such that genes farther from the telomere are less enriched for Sir3p (Fig. 5C). Comparison of the Sir3-TAP gradient of wild type and $h t z 1-K 3,8,10,14 R$ showed that Sir3p was present in greater levels and spread farther in the mutant. These data confirm the qRT-PCR results that indicated an inability of $h t z 1-K 3,8,10,14 R$ to block the spread of silent chromatin. These data identify the first specific function for H2A.Z acetylation in vivo. We next assayed the boundaries at $H M R$ by testing the expression levels of YCR095c and GIT1 (Fig. 5A). In contrast to the telomeres, the boundaries blocking the spread of silencing at $H M R$ were unaffected in the $h t z 1-K 3,8,10,14 R$. The lack of heterochromatic spreading in this context was likely due to the dual boundary mechanisms at work at this locus (Donze et al. 1999; Oki and Kamakaka 2005), as discussed below.

If acetylated H2A.Z were the chief contributor to the block in spreading of heterochromatin at telomeres, then a deficiency in NuA4 should lead to a similar boundary defect at the telomeres. Indeed, esa1-1851 cells were more severely defective in boundary function than either $h t z 1 \Delta$ or the unacetylatable form of H2A.Z at telomere IX-R (Fig. 5A). The more severe phenotype of the esa11851 cells was probably due to the combination of a twofold decrease in H2A.Z enrichment (Fig. 5C) and the decrease in acetylation of the remaining H2A.Z.

\section{H2A.Z acetylation was essential in stress conditions when NuA4 is compromised}

An $h t z 1 \Delta$ mutation is lethal in combination with null alleles of many genes involved in chromatin structure. These lethal combinations include mutations in genes involved in $\mathrm{H} 2 \mathrm{~B}$ ubiquitination (rad6s and bre1s), H3 methylation (set2 $\Delta$ ) (Krogan et al. 2003), and H4 acetylation (eaf1s) (Kobor et al. 2004). This trend was extended here by the lethality of the gcn $5 \Delta$ eaf $1 \Delta$ doublemutant combination, deficient in both SAGA and NuA4. Taken together, the various synthetic lethal interactions suggested that cells lacking certain chromatin modifications require $\mathrm{H} 2 \mathrm{~A} . \mathrm{Z}$ for viability, and that nucleosomes require a minimum amount of modification to support viability. We tested whether an unacetylatable H2A.Z would be lethal in combination with mutations in genes for known histone modifiers. Indeed, $h t z 1-K 3,8,10,14 R$ in combination with eaf1s, a nonessential subunit of $\mathrm{NuA} 4$, caused severe growth retardation, evident in the dilution series of a tetra-type tetrad from the cross (Fig. 6A). The double mutant was more impaired than either single mutant, especially at $37^{\circ} \mathrm{C}$. Although eaf $1 \Delta$ cells are somewhat compromised compared with wild type or $h t z 1-K 3,8,10,14 R$, at this temperature the double mutants were not viable. The simplest interpretation of the double-mutant phenotype was that acetylation of either H2A.Z or some other NuA4 target, but not both, was essential to maintain cell viability. These data also suggest that the presence of $\mathrm{H} 2 \mathrm{~A} . \mathrm{Z}$ is not sufficient to pro- 

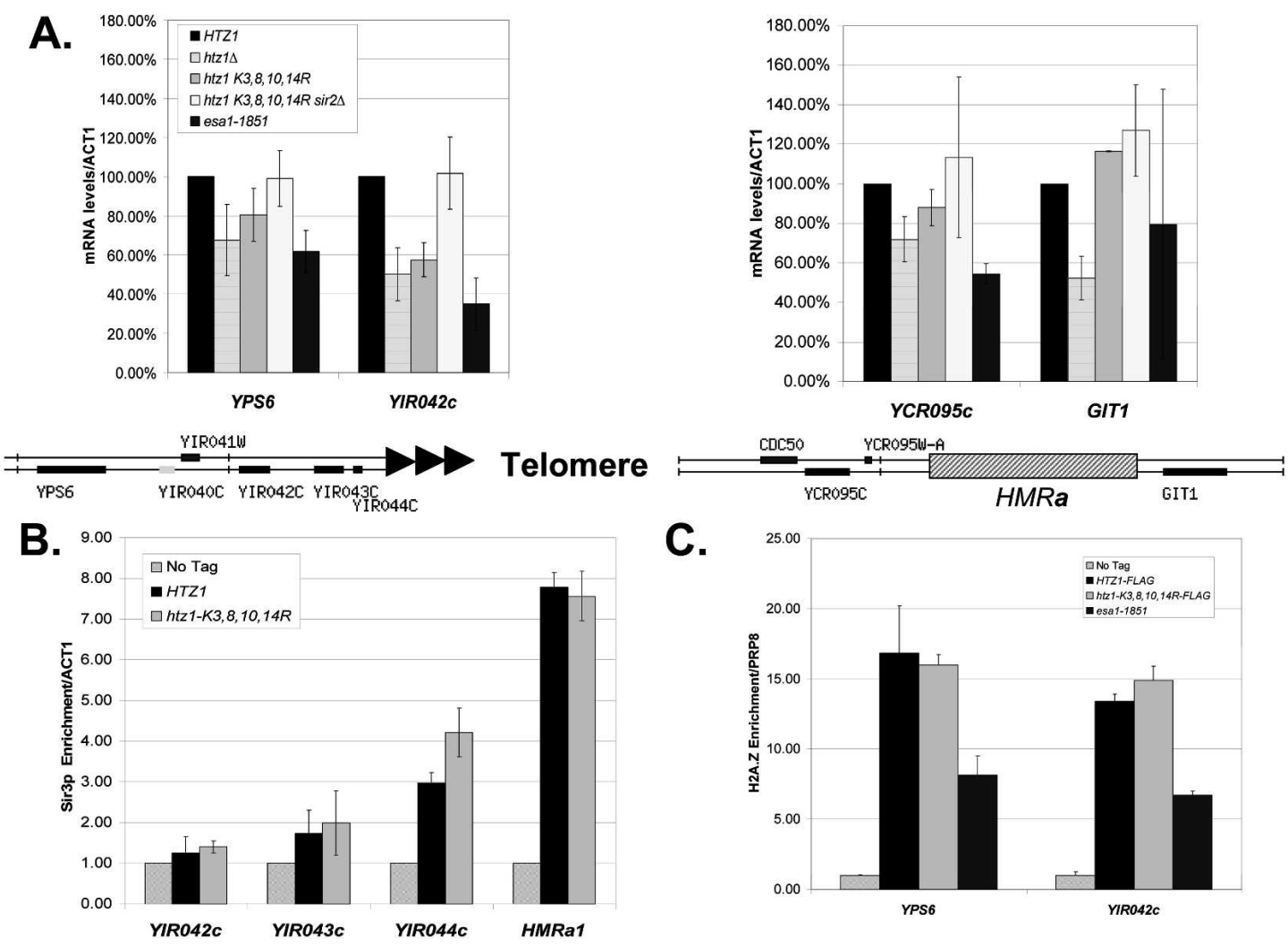

Figure 5. H2A.Z acetylation was important for a boundary to silent chromatin. $(A)$ qRT-PCR of a gene cluster near the telomere of chromosome IX-R and the genes adjacent to the left and right boundaries of $H M R$. (B) ChIP analysis of Sir3p at chromosome IX-R. (C) ChIP analysis of H2A.Z at chromosome IX-R described in $A$. Error bars represent standard deviation between four independent RNA purifications $(A)$ or two independent $\operatorname{ChIPs}(B, C)$.

vide full function; rather, modified forms of H2A.Z must be present.

\section{H2A.Z or H4 acetylation provided an essential function}

To explore the inviability of cells that could not acetylate H2A.Z, we tested double mutants containing an unacetylatable form of H2A.Z with unacetylatable forms of NuA4 targets, H2A and H4. To test whether acetylated $\mathrm{H} 4$ provided the essential function in cells lacking acetylated H2A.Z, a strain was constructed that carried a wild-type version of an $\mathrm{H} 4$ gene (HHF2) on a plasmid whose loss could be selected for on medium containing 5 -fluoro-orotic acid (FOA). This strain contained an allele of HTZ1 that encoded an unacetylatable H2A.Z (htz1-K3,8,10,14R) as well as a mutant version of $\mathrm{H} 4$ that could not be acetylated on the positions recognized by NuA4 (hhf2-K5,8,12R). The second copy of the histone H4 gene (HHF1) was deleted. This strain could not grow in the absence of the wild-type $\mathrm{H} 4$ gene as seen by the lack of growth on FOA-containing medium (Fig. 6B). Hence, growth required acetylated versions of either H2A.Z or H4. Moreover, since H2A was wild type in these cells, it could not provide an equivalent essential function of $\mathrm{H} 4$. This was confirmed by combining the unacetylatable H2A.Z (htz1-K3,8,10,14R) and unacety- latable $\mathrm{H} 2 \mathrm{~A}$ (hta1-K4,7R) and finding that the double mutants were fully viable (data not shown). Likewise, acetylation of $\mathrm{H} 4 \mathrm{~K} 16$ by the SAS HAT complex was unable to provide this function, as sas $2 \Delta$ htz1$K 3,8,10,14 R$ cells had no discernable growth defect (data not shown).

\section{Acetylation was unimportant for some roles of H2A.Z}

The htz1-K3,8,10,14R mutant allowed us to determine if H2A.Z had roles other than serving as a boundary to silent chromatin. One striking property of $h t z 1 \Delta \mathrm{mu}-$ tants is their sensitivity to a variety of chemicals that cause genotoxic and perhaps other stresses to cells. We assayed the influence of acetylation on the resistance of cells to these chemicals. $h t z 1-K 3,8,10,14 R$, as well as individual point mutants, grew as well as wild type on media containing hydroxyurea, benomyl, formamide, and caffeine (data not shown). esa1-1851 cells are sensitive to methyl methanesulfonate and camptothecin (Bird et al. 2002), yet the unacetylatable H2A.Z mutant also grew as well as wild type under these stresses. The similarity in sequence between canonical H2A and H2A.Z, as well as NuA4's ability to acetylate both, led to the obvious possibility that acetylation of $\mathrm{H} 2 \mathrm{~A}$ and of H2A.Z had overlapping roles. To test whether the lack of chemical sensitivity of the $h t z 1-K 3,8,10,14 R$ mutant was due 


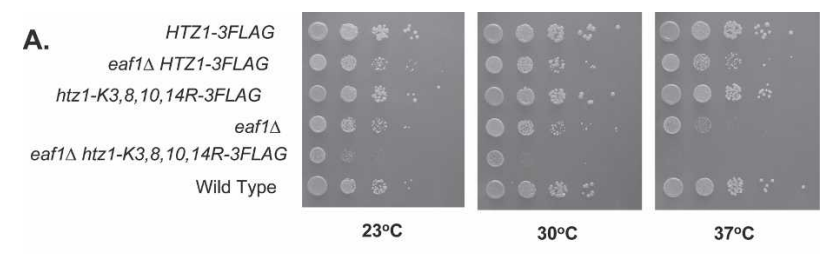

B.

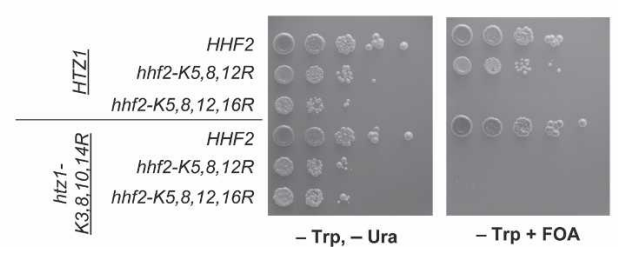

Figure 6. Genetic interactions between HTZ1 alleles and mutations affecting histone $\mathrm{H} 4$ acetylation. (A) htz1-K3, 8, 10,14R3Flag:: KanMX was crossed to eaf1 $1 \Delta:$ His $3 M X$, and tetrads were analyzed. Tenfold serial dilutions were spotted on minimal media and grown for $3 \mathrm{~d}$ at the indicated temperatures. $(B)$ Wildtype HHF2 was carried on a URA3 plasmid in a background with either wild-type HTZ1 or htz1-K3,8,10,14R-3Flag. These strains were transformed with either wild-type HHF2, hhf2$K 5,8,12 R$, or $h h f 2-K 5,8,12,16 R$ on a TRP1 plasmid. Cells were grown in medium containing tryptophan. Tenfold serial dilutions were spotted on either minimal medium lacking tryptophan and lacking uracil or minimal medium lacking tryptophan and containing FOA, to select against the URA3 plasmid. Cells were grown for $3 \mathrm{~d}$ at $23^{\circ} \mathrm{C}$ prior to imaging.

to acetylation of $\mathrm{H} 2 \mathrm{~A}$ by $\mathrm{NuA} 4$, the $h t z 1-K 3,8,10,14 R$ mutation was combined with a version of $\mathrm{H} 2 \mathrm{~A}$ that cannot be acetylated (hta1-K4,7R). This created a double mutant with no version of either H2A or H2A.Z that could be acetylated by NuA4. However, on media containing all of the drugs described above, there was no discernable growth difference between either the individual or the double mutant from wild type (data not shown). Therefore, it was H2A.Z itself, and not its acetylated forms, that conferred resistance to these chemical agents.

\section{Discussion}

This work focused on whether the H2A variant H2A.Z was subjected to covalent modification, and if so, by which enzymes, and for what purpose. The results revealed that acetylated H2A.Z contributed to the boundaries between euchromatin and heterochromatin, and played an unanticipated role essential for viability.

\section{H2A.Z acetylation: the interplay of SRW1-Com, NuA4, and SAGA}

The results presented here established that $\mathrm{H} 2 \mathrm{~A} . \mathrm{Z}$ was a substrate for the HAT NuA4 in vitro and in vivo. Moreover, H2A.Z purified from yeast cells was acetylated in vivo at multiple sites. The majority of H2A.Z modifications depended on the HAT NuA4, and at least one depended on SAGA (GCN5). NuA4 acetylation of H2A.Z was a prerequisite for SAGA acetylation, since esa1-
L357H and esa1-L357H gcn5s were equally deficient in acetylated species. Analysis of mutations of individual lysine residues in H2A.Z's N-terminal tail established that all four $\mathrm{N}$-terminal lysines are capable of being acetylated.

The relative lack of acetylation of H2A.Z in the swr1s mutant suggested that H2A.Z must be incorporated into nucleosomes by SWR1-Com for subsequent acetylation by NuA4. This conclusion was confirmed by evaluating the modification status of soluble versus chromatin-associated H2A.Z: H2A.Z was mostly unmodified prior to deposition and became appropriately modified postdeposition. These results eliminated the possibility that H2A.Z acetylation was a requirement for its recognition by, or association with, SWR1-Com and therefore deposition. Taken together, these data suggest a model (Fig. 7) in which SWR1-Com deposits an unmodified H2A.Z, presumably in the form of an H2A.Z-H2B dimer that is exchanged for an H2A-H2B dimer. Following deposition, $\mathrm{NuA} 4$ is recruited to the unmodified H2A.Z and adds one or more acetyl groups. Following NuA4 acetylation, SAGA may add a final acetyl group.

Initially, we considered the possibility that the subunits shared between SWR1-Com and NuA4 was a coincidence, but results presented here supported more interesting possibilities. Specifically, the hypoacetylation of the fraction of H2A.Z incorporated into chromatin in the absence of SWR1-Com indicated that chromatin association was not sufficient to ensure efficient acetylation by NuA4. This result suggested coupling between SWR1-Com and NuA4 vis-à-vis H2A.Z, with intriguing possible mechanisms for such a coupling. The shared subunits between SWR1-Com and NuA4 may remain associated with H2A.Z, with the unshared subunits of the two complexes exchanging partners. In support of coupling deposition and acetylation, deletion of YAF9, a nonessential subunit shared between SWR1-Com and $\mathrm{NuA} 4$, results in a reduction in both H2A.Z deposition and $\mathrm{H} 4$ acetylation near the telomere (Zhang et al. 2004). A simple interpretation of these results is that when H2A.Z is not correctly deposited, NuA4 is not recruited, and hence sites of NuA4 acetylation on $\mathrm{H} 2 \mathrm{~A}$ and $\mathrm{H} 4$ remain hypoacetylated. It will be interesting to determine whether H2A.Z recognition by SWR1-Com and $\mathrm{NuA} 4$ is mediated by the shared subunits.

An interesting clue as to why H2A.Z deposition and acetylation might be coupled was suggested by the discovery that H2A.Z localization was reduced by half in an esa1-1851 mutant when compared with wild type. Since SWR1-Com is an enzyme that exchanges H2A-H2B dimers for H2A.Z-H2B dimers, in the absence of a sub-

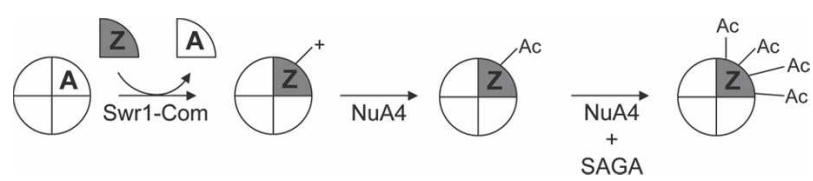

Figure 7. A model linking SWR1-Com and NuA4 in H2A.Z function. Following deposition into chromatin, H2A.Z is first acetylated by NuA4 and subsequently by NuA4 and SAGA. 
stantial change in free energy, one would expect the reverse reaction to also be catalyzed by SWR1-Com. Perhaps acetylation of H2A.Z- containing nucleosomes by NuA4 disfavors the reverse reaction. If so, the lack of $\mathrm{H} 2 \mathrm{~A} . \mathrm{Z}$ and/or $\mathrm{H} 4$ acetylation in these nucleosomes may allow SWR1-Com to remove H2A.Z-H2B dimers, resulting in a reduced level of $\mathrm{H} 2 \mathrm{~A} . \mathrm{Z}$ in chromatin. A decisive test of this model will require kinetic analysis of H2A.Z deposition in wild-type and NuA4 mutant strains.

\section{H2A. $Z$ acetylation and chromatin boundary function}

A heterochromatin boundary function for H2A.Z acetylation was suggested by the esa1-L254P mutation causing a decrease in the expression of telomere-proximal genes (Zhang et al. 2004) combined with the discovery here that H2A.Z is a substrate of NuA4. Indeed, an unacetylatable H2A.Z was defective in boundary function at the telomeres, even though localization of the mutant H2A.Z was indistinguishable from wild type. In addition, expression levels of genes that were repressed in the $h t z 1-K 3,8,10,14 R$ mutant were restored in the absence of Sir2p. This inference was confirmed by directly assaying Sir3p levels at these chromosomal locations in wild-type and $h t z 1-K 3,8,10,14 R$ mutants and finding that Sir3p spread beyond its normal boundaries in the mutant. Thus, acetylation of H2A.Z was important in restricting the spread of Sir proteins from telomeres into subtelomeric positions. However, boundaries of silenced chromatin at $H M R$ did not depend on acetylation of H2A.Z. One boundary at $H M R$ corresponds to a flanking tRNA gene whose promoter seems to play a critical role in the function of the boundary (Donze et al. 1999; Oki and Kamakaka 2005). Mutations in HAT genes do not result in heterochromatin spreading beyond this boundary. Only when the tRNA gene is deleted in combination with a HAT mutant do the Sir proteins spread. Presumably the $h t z 1-K 3,8,10,14 R$ mutation in combination with deletion of the tRNA gene would result in Sir protein spreading beyond this boundary.

The fact that H2A.Z acetylation prevents spreading of silencing raises the intriguing possibility that acetylated H2A.Z modulates a chromatin structure that is incompatible with Sir protein binding and hence with silencing. Indeed, nucleosomal arrays containing Drosophila H2A.Z prevent the formation of highly condensed heterochromatic structures that are incompatible with transcription (Fan et al. 2002). If this model is correct, then acetylated tails of $\mathrm{H} 2 \mathrm{~A} . \mathrm{Z}$ rather than interactions between octamer cores would be the critical limitation to forming these specialized structures. In a similar spirit, a protein could specifically bind acetylated H2A.Z and block the spread of Sir proteins. Such a protein would be unable to bind the $h t z 1-K 3,8,10,14 R$ mutant, leading to the ectopic spread of the Sir proteins. Mutations in this protein should result in similar defects in the boundary of heterochromatin. Finally, acetylated tails of H2A.Z may be molecular decoys that can be recognized by and inhibit the Sir2p deacetylase.
Essential and nonessential roles for H2A.Z in Saccharomyces

A common genetic misconception is that genes in which null alleles are viable do not encode essential functions. Essential functions may be shared by overlapping proteins. The latter case seems to apply broadly to H2A.Z, as revealed by many synthetic lethal double-mutant combinations (A. Allison and M. Smith, pers. comm.). This study uncovered an essential function specific to the acetylated form of $\mathrm{H} 2 \mathrm{~A} . \mathrm{Z}$ that in combination with eaf $1 \Delta$ was severely growth-impaired at $23^{\circ} \mathrm{C}$ and lethal at $37^{\circ} \mathrm{C}$. The essential function provided by acetylated H2A.Z could also be fulfilled by acetylated H4 but not by acetylated $\mathrm{H} 2 \mathrm{~A}$. At present, the difference between the ability of $\mathrm{H} 2 \mathrm{~A}$ and $\mathrm{H} 4$ to carry out an essential function shared with H2A.Z could reflect either qualitative or quantitative differences between the two proteins. There are only two possible sites for acetylation of $\mathrm{H} 2 \mathrm{~A}$, and four on H2A.Z and H4. Hence, cells might need a minimum amount of nucleosome acetylation for viability. However, since H2A.Z is only $10 \%$ as abundant as $\mathrm{H} 2 \mathrm{~A}$, qualitative differences between $\mathrm{H} 2 \mathrm{~A}$ and $\mathrm{H} 4$ seem more likely. Interestingly, no NuA4 mutant has been recovered that completely abolishes H2A.Z acetylation. Presumably the last remaining acetyl group is important for robust growth when $\mathrm{H} 4$ acetylation is also reduced.

\section{Materials and methods}

Yeast strains and plasmids

All yeast strains are in the W303 background and are listed in Table 1. One-step integration of knockout cassettes (Longtine et al. 1998; Goldstein and McCusker 1999) and the C-terminal Flag-tag (Gelbart et al. 2001) have been previously described (see Supplementary Table 1 for primer sequences). HTZ1 was cloned into pRS316 using gap-repair, resulting in plasmid pJR2658. HTZ1 was amplified from genomic DNA using Pfu-Ultra (Stratagene) (the primers are listed in Supplementary Table 1). The purified PCR product was cotransformed into JRY7754 (htz1s::His3MX) with pRS316 digested with HindIII and BamHI. Plasmids were rescued from $\mathrm{Ura}^{+}$transformants, and the integrity of the insert was verified by DNA sequencing. pJR2658 was transformed into JRY7754 (htz1D::HIS3MX). A C-terminal 3Flag-tag was integrated on the plasmid in frame with the HTZ1 open reading frame and verified by DNA sequencing, resulting in pJR2959. Site-directed mutagenesis was carried out using Quikchange XL (Stratagene) on pJR2969 mutating K3R (pJR2970), K8R (pJR2971), K10R (pJR2972), K14R (pJR2973), and K3,8,10,14R (pJR2974) (see Supplementary Table 1 for primers). htz1-K3,8,10,14R-3Flag::KanMX was integrated at its chromosomal locus by amplifying the gene from the plasmid using Pfu-Ultra and transforming a strain $h t z 1 \Delta:: U R A 3 M X$ (JRY7970) and selecting for G418 resistance. G418-resistant cells were then screened for FOA resistance, to identify cells that had gained the gained the $h t z 1$ multiple site mutant at the HTZ1 locus and lost the URA3 insertion at that locus. The HTZ1 locus from strains that were resistant to both drugs was PCR-amplified using Pfu-Ultra and sequenced.

\section{Protein purifications}

S. cerevisiae canonical histones were expressed individually in E. coli and purified from inclusion bodies as described (Luger et 
Table 1. Yeast strains

\begin{tabular}{|c|c|c|}
\hline Strain & Genotype & Source \\
\hline W303-1a & MATa ade2-1 leu2-3, 112 his3-1 ura3-52 trpl-100 can1-100 & R. Rothstein \\
\hline JRY7721 & MATa $E S A 1-T A P:: T R P 1$ & Kobor et al. 2004 \\
\hline JRY7754 & MAT $\boldsymbol{\alpha} h t z 1 \Delta:: H I S 13 M X$ & Kobor et al. 2004 \\
\hline JRY7763 & MAT $\boldsymbol{\alpha}$ eaf $1 \Delta:: H I S 3 M X$ & Kobor et al. 2004 \\
\hline JRY7968 & MAT $\boldsymbol{\alpha}$ esa1-1851::URA3 & Bird et al. 2002 \\
\hline JRY7969 & MAT $\boldsymbol{\alpha}$ esa1-L357H::URA3 & Bird et al. 2002 \\
\hline JRY7970 & MATa $h t z 1 \Delta:: U R A 3 M X$ & This study \\
\hline JRY7971 & MATa HTZ1-3Flag::KanMX & This study \\
\hline JRY7972 & MAT $\boldsymbol{\alpha}$ HTZ1-3Flag:: KanMX & This study \\
\hline JRY7973 & MATa esa1-1851::URA3 HTZ1-3Flag::KanMX & This study \\
\hline JRY7974 & MAT $\boldsymbol{\alpha}$ esa1-1851::URA3 HTZ1-3Flag::KanMX & This study \\
\hline JRY7975 & MATa eaf1D::HIS3MX HTZ1-3Flag::KanMX & This study \\
\hline JRY7976 & MATa swf1D::HIS3MX HTZ1-3Flag::KanMX & This study \\
\hline JRY7977 & MATa gcn $5 \Delta:: H I S 3 M X$ HTZ1-3Flag::KanMX & This study \\
\hline JRY7978 & MATa sas2D::HIS3MX HTZ1-3Flag::KanMX & This study \\
\hline JRY7979 & MATa esa1-L357H::URA3 HTZ1-3Flag::KanMX & This study \\
\hline JRY7980 & MATa esa1-L357H:: URA3 gcn5s:: HIS3MX HTZ1-3Flag::KanMX & This study \\
\hline JRY7981 & MATa $h t z 1 \Delta:: H I S 3 M X$ [pRS314 HTZ1-3Flag::KanMX] & This study \\
\hline JRY7982 & MATa $h t z 1 \Delta:: H I S 3 M X[p R S 314 h t z 1 K 3,8,10,14 R-3 F l a g::$ KanMX] & This study \\
\hline JRY7983 & MAT $\boldsymbol{\alpha} h t z 1-K 3,8,10,14 R-3 F l a g:: K a n M X$ & This study \\
\hline JRY7984 & MAT $\boldsymbol{\alpha} h t z 1-K 3,8,10,14 R-3 F l a g:: K a n M X$ sir2s ::His3MX & This study \\
\hline JRY7985 & MATa $h t z 1 \Delta:: H I S 3 M X$ [pRS314 htz1K3R-3Flag::KanMX] & This study \\
\hline JRY7986 & MATa $h t z 1 \Delta:: H I S 3 M X[p R S 314 h t z 1 K 8 R-3 F l a g:: K a n M X]$ & This study \\
\hline JRY7987 & MATa $h t z 1 \Delta:: H I S 3 M X[p R S 314$ htz1K10R-3Flag::KanMX] & This study \\
\hline JRY7988 & MATa $h t z 1 \Delta:: H I S 3 M X[p R S 314 h t z 1$ K14R-3Flag::KanMX] & This study \\
\hline JRY7989 & MAT $\boldsymbol{\alpha}(h h f 1-h h t 1) \Delta:: H p h M X(h h f 2-h h t 2) \Delta:: N a t M X[p R S 316$ HHF2-HHT2] & This study \\
\hline JRY7990 & $\begin{array}{l}\text { MAT } \boldsymbol{\alpha}(h h f 1-h h t 1) \Delta:: H p h M X(h h f 2-h h t 2) \Delta:: \text { NatMX htz1-K3,8,10,14R-3Flag ::KanMX } \\
\text { [pRS316 HHF2-HHT2] }\end{array}$ & This study \\
\hline JRY8121 & MAT $\boldsymbol{\alpha}$ HTZ1-3Flag::KanMX SIR3-TAP::TRP1 & This study \\
\hline JRY8122 & MAT $\boldsymbol{\alpha}$ htz1-K3,8,10,14R-3Flag::KanMX SIR3-TAP::TRP1 & This study \\
\hline
\end{tabular}

al. 1999; Dyer et al. 2004). N-terminally tagged Htzlp with a six-histidine epitope was expressed in E. coli, and inclusion bodies containing recombinant proteins were purified as described. After denaturing the inclusion bodies, $\mathrm{His}_{6}-\mathrm{Htzl} \mathrm{p}$ was purified using Talon Cobalt Affinity Resin (BD Biosciences) and eluted with $300 \mathrm{mM}$ Imidazole. Reconstitution of octamers containing canonical H2A, or H2A.Z, was done as described (Luger et al. 1999; Dyer et al. 2004). Untagged H2A.Z for mass spectrometry was purified as described for canonical H2A (Luger et al. 1999).

Native NuA4 complex was purified from $6 \mathrm{~L}$ of $S$. cerevisiae containing Esalp-TAP. TAP purifications were carried out as described, except the buffer was 40 mM HEPES (pH 7.3), 350 $\mathrm{mM} \mathrm{NaCl}, 0.1 \%$ Tween-20, $10 \%$ glycerol, $5 \mathrm{mM}$ 2-mercaptoethanol, and $1 \times$ Complete Protease Inhibitor Cocktail (Roche), and the TEV cleavage was performed overnight at $4{ }^{\circ} \mathrm{C}$ (Kobor et al. 2004).

\section{Histone acetylation assays}

HAT assays were preformed as described (Allard et al. 1999). ${ }^{3} \mathrm{H}$-acetyl-CoA $(0.125 \mu \mathrm{Ci})$ was added to $10 \mu \mathrm{L}$ of $\mathrm{NuA} 4$ along with varying amounts of wild-type dimers or octamers, and H2A.Z dimers or octamers in HAT buffer $(50 \mathrm{mM}$ Tris- $\mathrm{HCl}$ at pH 8.0, 50 mM KCl, 0.1 mM EDTA, 5\% glycerol, 1 mM dithiothreitol [DTT], $1 \mathrm{mM}$ phenylmethylsulfonyl fluoride [PMSF], 10 $\mathrm{mM}$ sodium butyrate) for $30 \mathrm{~min}$ at $30^{\circ} \mathrm{C}$. For nucleosome acetylation assays, H2A.Z-containing nucleosomes were purified as described below for the chromatin association assay. Following $\alpha$ Flag affinity purification, $25 \mu \mathrm{L}$ of NuA4 was added in HAT buffer with $0.125 \mu \mathrm{Ci}$ of ${ }^{3} \mathrm{H}$-acetyl-CoA for $30 \mathrm{~min}$ at $30^{\circ} \mathrm{C}$. Sample buffer was added, and the samples were loaded onto a $15 \%$ SDS-polyacrylamide gel. Following electrophoresis, the gel was transferred to nitrocellulose and exposed to film using a Biomax Transcreen LE (Kodak).

\section{AU gels}

Cells were grown to mid-log phase, and $100 \mathrm{OD}_{600}$ units were harvested by centrifugation. Cells were resuspended in Buffer HIP (50 mM Tris-Cl at pH 7.8, $200 \mathrm{mM} \mathrm{NaCl}, 1.5 \mathrm{mM} \mathrm{MgAc}$, $100 \mathrm{mM}$ Na Butyrate, $5 \mathrm{mM}$ Nicotinamide, $10 \mathrm{mM}$ NaPPi, 0.1 $\mathrm{mM} \mathrm{Na}_{3} \mathrm{VO}_{4}, 5 \mathrm{mM} \mathrm{NaF}$, Complete Protease inhibitor cocktail [Roche]). Acid-washed glass beads were added, and the cells were disrupted mechanically using a bead beater (BioSpec Products) for $5 \mathrm{~min}$. $\mathrm{CaCl}_{2}$ was added to a final concentration of 1 $\mathrm{mM}$, and chromosomal DNA was digested using $10 \mathrm{U}$ of micrococcal nuclease (Sigma) for $10 \mathrm{~min}$ at $37^{\circ} \mathrm{C}$. Insoluble material was removed by centrifugation. The supernatant was removed and incubated with $25 \mu \mathrm{L}$ of $\alpha$ Flag M2 beads (Sigma) for $90 \mathrm{~min}$ at $4^{\circ} \mathrm{C}$. Beads were then pelleted and washed three times with $0.6 \mathrm{~mL}$ of Buffer HIP. After washing, the supernatant was completely removed by aspiration with a 30 -gauge needle. The dry beads were resuspended in AU gel loading buffer (6 M urea, $5 \%$ acetic acid, $0.02 \%$ Pyronin Y). AU gels were set up and run as described, except Triton X-100 was omitted (Lennox and Cohen 1989). The gel was transferred to a PVDF membrane (Millipore) in $0.9 \%$ acetic acid toward the cathode (Rogakou et al. 2000) and immunoblotted using Rabbit $\alpha$ Flag (Sigma), followed by secondary anti-rabbit IgG conjugated to Alexa Fluor 680 (Invitrogen). The immunoblot was scanned using a LiCor Odyssey Infrared Imaging System. 


\section{Chromatin association assay}

The chromatin association assay was carried out essentially as described, except $100 \mathrm{mM} \mathrm{Na}$ butyrate and $5 \mathrm{mM}$ nicotinamide were added to inhibit histone deacetylases (Donovan et al. 1997; Liang and Stillman 1997). The soluble fractions and MNasedigested fractions were affinity-purified using $\alpha$ Flag M2 beads (Sigma) and subjected to AU gel analysis as described above.

\section{Quantitative PCR}

RNA was purified from $S$. cerevisiae using the hot-phenol method (Schmitt et al. 1990). RNA was digested with RNasefree DNase I (Roche and Qiagen). cDNA was synthesized using SuperScript III First-Strand Synthesis System for RT-PCR and oligo(dT) (Invitrogen). cDNA was analyzed using an Mx3000P qPCR system (Stratagene) and SYBR green core reagents (Applied Biosystems). ACT1 cDNA served as a loading control. Samples were analyzed in triplicate for two independent RNA preparations. The primer sequences are listed in Supplementary Table 2.

ChIP was performed as described (Davies et al. 2005). IPs of H2A.Z were performed using $\alpha$ Flag M2 affinity resin (Sigma), and IPs of Sir3p-TAP were performed using IgG resin (Amersham). Input DNA and ChIP DNA were analyzed using an Mx3000P qPCR system (Stratagene) and SYBR green core reagents (Applied Biosystems). Samples were analyzed in triplicate for two independent ChIPs. The primer sequences are listed in Supplementary Table 2.

\section{Acknowledgments}

We dedicate this paper to the memory of our friend and colleague John Newport from University of California at San Diego, who contributed so much to what we know of nuclear biology. We are grateful to Paul Kaufman, Toshio Tsukiyama, Oliver Rando, and Mitch Smith for strains and reagents. We also thank Mike Botchan, Barbara Meyer, and Jake Mayfield for critical discussion. We are especially grateful to Mike Kobor for help and inspiration throughout this project. We thank Michael Keogh and Steve Buratowski for communications prior to publication. We also thank David King and Arnold Falick for mass spectrometry analysis. J.E.B. was supported by NIH training grants (5 T32 GM07232 and 5 T32 ES07075). J.E.H. was supported by a predoctoral fellowship from the National Science Foundation. This work was supported by NIH grant GM31105.

\section{References}

Allard, S., Utley, R.T., Savard, J., Clarke, A., Grant, P., Brandl, C.J., Pillus, L., Workman, J.L., and Cote, J. 1999. NuA4, an essential transcription adaptor/histone $\mathrm{H} 4$ acetyltransferase complex containing Esalp and the ATM-related cofactor Tralp. EMBO J. 18: 5108-5119.

Bird, A.W., Yu, D.Y., Pray-Grant, M.G., Qiu, Q., Harmon, K.E., Megee, P.C., Grant, P.A., Smith, M.M., and Christman, M.F. 2002. Acetylation of histone $\mathrm{H} 4$ by Esal is required for DNA double-strand break repair. Nature 419: 411-415.

Carmen, A.A., Milne, L., and Grunstein, M. 2002. Acetylation of the yeast histone $\mathrm{H} 4 \mathrm{~N}$ terminus regulates its binding to heterochromatin protein SIR3. I. Biol. Chem. 277: 47784781.

Chakravarthy, S., Bao, Y., Roberts, V.A., Tremethick, D., and Luger, K. 2004. Structural characterization of histone H2A variants. Cold Spring Harb. Symp. Quant. Biol. 69: 227-234.

Clarke, A.S., Lowell, J.E., Jacobson, S.J., and Pillus, L. 1999.
Esalp is an essential histone acetyltransferase required for cell cycle progression. Mol. Cell. Biol. 19: 2515-2526.

Davies, B.S., Wang, H.S., and Rine, J. 2005. Dual activators of the sterol biosynthetic pathway of Saccharomyces cerevisiae: Similar activation/regulatory domains but different response mechanisms. Mol. Cell. Biol. 25: 7375-7385.

Dion, M.F., Altschuler, S.J., Wu, L.F., and Rando, O.J. 2005. Genomic characterization reveals a simple histone $\mathrm{H} 4$ acetylation code. Proc. Nat1. Acad. Sci. 102: 5501-5506.

Donovan, S., Harwood, J., Drury, L.S., and Diffley, J.F. 1997. Cdc6p-dependent loading of $\mathrm{Mcm}$ proteins onto pre-replicative chromatin in budding yeast. Proc. Natl. Acad. Sci. 94: 5611-5616.

Donze, D., Adams, C.R., Rine, J., and Kamakaka, R.T. 1999. The boundaries of the silenced HMR domain in Saccharomyces cerevisiae. Genes \& Dev. 13: 698-708.

Doyon, Y. and Cote, J. 2004. The highly conserved and multifunctional NuA4 HAT complex. Curr. Opin. Genet. Dev. 14: $147-154$.

Doyon, Y., Selleck, W., Lane, W.S., Tan, S., and Cote, J. 2004. Structural and functional conservation of the NuA4 histone acetyltransferase complex from yeast to humans. Mol. Cell. Biol. 24: 1884-1896.

Dyer, P.N., Edayathumangalam, R.S., White, C.L., Bao, Y., Chakravarthy, S., Muthurajan, U.M., and Luger, K. 2004. Reconstitution of nucleosome core particles from recombinant histones and DNA. Methods Enzymol. 375: 23-44.

Fan, J.Y., Gordon, F., Luger, K., Hansen, J.C., and Tremethick, D.J. 2002. The essential histone variant H2A.Z regulates the equilibrium between different chromatin conformational states. Nat. Struct. Biol. 9: 172-176.

Gelbart, M.E., Rechsteiner, T., Richmond, T.J., and Tsukiyama, T. 2001. Interactions of Isw 2 chromatin remodeling complex with nucleosomal arrays: Analyses using recombinant yeast histones and immobilized templates. Mol. Cell. Biol. 21: 2098-2106.

Goldstein, A.L. and McCusker, J.H. 1999. Three new dominant drug resistance cassettes for gene disruption in Saccharomyces cerevisiae. Yeast 15: 1541-1553.

Kamakaka, R.T. and Biggins, S. 2005. Histone variants: Deviants? Genes \& Dev. 19: 295-310.

Kimura, A., Umehara, T., and Horikoshi, M. 2002. Chromosomal gradient of histone acetylation established by Sas $2 \mathrm{p}$ and Sir2p functions as a shield against gene silencing. Nat. Genet. 32: 370-377.

Kobor, M.S., Venkatasubrahmanyam, S., Meneghini, M.D., Gin, J.W., Jennings, J.L., Link, A.J., Madhani, H.D., and Rine, J. 2004. A protein complex containing the conserved Swi2/ Snf2-related ATPase Swrlp deposits histone variant H2A.Z into euchromatin. PLOS Biol. 2: E131.

Krogan, N.J., Keogh, M.C., Datta, N., Sawa, C., Ryan, O.W., Ding, H., Haw, R.A., Pootoolal, J., Tong, A., Canadien, V., et al. 2003. A Snf2 family ATPase complex required for recruitment of the histone H2A variant Htz1. Mol. Cell 12: 15651576.

Krogan, N.J., Baetz, K., Keogh, M.C., Datta, N., Sawa, C., Kwok, T.C., Thompson, N.J., Davey, M.G., Pootoolal, J., Hughes, T.R., et al. 2004. Regulation of chromosome stability by the histone H2A variant Htz1, the Swr1 chromatin remodeling complex, and the histone acetyltransferase NuA4. Proc. Nat1. Acad. Sci. 101: 13513-13518.

Kusch, T., Florens, L., Macdonald, W.H., Swanson, S.K., Glaser, R.L., Yates III, J.R., Abmayr, S.M., Washburn, M.P., and Workman, J.L. 2004. Acetylation by Tip60 is required for selective histone variant exchange at DNA lesions. Science 306: 2084-2087. 
Lennox, R.W. and Cohen, L.H. 1989. Analysis of histone subtypes and their modified forms by polyacrylamide gel electrophoresis. Methods Enzymol. 170: 532-549.

Liang, C. and Stillman, B. 1997. Persistent initiation of DNA replication and chromatin-bound MCM proteins during the cell cycle in cdc6 mutants. Genes \& Dev. 11: 3375-3386.

Longtine, M.S., McKenzie III, A., Demarini, D.J., Shah, N.G., Wach, A., Brachat, A., Philippsen, P., and Pringle, J.R. 1998. Additional modules for versatile and economical PCR-based gene deletion and modification in Saccharomyces cerevisiae. Yeast 14: 953-961.

Luger, K., Rechsteiner, T.J., and Richmond, T.J. 1999. Expression and purification of recombinant histones and nucleosome reconstitution. Methods Mol. Biol. 119: 1-16.

Meneghini, M.D., Wu, M., and Madhani, H.D. 2003. Conserved histone variant H2A.Z protects euchromatin from the ectopic spread of silent heterochromatin. Cell 112: 725-736.

Mizuguchi, G., Shen, X., Landry, J., Wu, W.H., Sen, S., and Wu, C. 2004. ATP-driven exchange of histone H2AZ variant catalyzed by SWR1 chromatin remodeling complex. Science 303: 343-348.

Oki, M. and Kamakaka, R.T. 2005. Barrier function at HMR. Mol. Cell 19: 707-716.

Peterson, C.L. and Laniel, M.A. 2004. Histones and histone modifications. Curr. Biol. 14: R546-R551.

Redon, C., Pilch, D., Rogakou, E., Sedelnikova, O., Newrock, K., and Bonner, W. 2002. Histone H2A variants H2AX and H2AZ. Curr. Opin. Genet. Dev. 12: 162-169.

Ren, Q. and Gorovsky, M.A. 2001. Histone H2A.Z acetylation modulates an essential charge patch. Mol. Cell 7: 1329-1335.

- 2003. The nonessential H2A N-terminal tail can function as an essential charge patch on the H2A.Z variant $\mathrm{N}$ terminal tail. Mol. Cell. Biol. 23: 2778-2789.

Rogakou, E.P., Redon, C., Boon, C., Johnson, K., and Bonner, W.M. 2000. Rapid histone extraction for electrophoretic analysis. Biotechniques 28: 38-40, 42, 46.

Rusche, L.N., Kirchmaier, A.L., and Rine, J. 2003. The establishment, inheritance, and function of silenced chromatin in Saccharomyces cerevisiae. Annu. Rev. Biochem. 72: 481516.

Santisteban, M.S., Kalashnikova, T., and Smith, M.M. 2000. Histone H2A.Z regulates transcription and is partially redundant with nucleosome remodeling complexes. Cell 103: 411-422.

Sarma, K. and Reinberg, D. 2005. Histone variants meet their match. Nat. Rev. Mol. Cell Biol. 6: 139-149.

Schmitt, M.E., Brown, T.A., and Trumpower, B.L. 1990. A rapid and simple method for preparation of RNA from Saccharomyces cerevisiae. Nucleic Acids Res. 18: 3091-3092.

Smith, E.R., Eisen, A., Gu, W., Sattah, M., Pannuti, A., Zhou, J., Cook, R.G., Lucchesi, J.C., and Allis, C.D. 1998. ESA1 is a histone acetyltransferase that is essential for growth in yeast. Proc. Natl. Acad. Sci. 95: 3561-3565.

Strahl, B.D. and Allis, C.D. 2000. The language of covalent histone modifications. Nature 403: 41-45.

Suka, N., Suka, Y., Carmen, A.A., Wu, J., and Grunstein, M. 2001. Highly specific antibodies determine histone acetylation site usage in yeast heterochromatin and euchromatin. Mol. Cell 8: 473-479.

Suka, N., Luo, K., and Grunstein, M. 2002. Sir2p and Sas2p opposingly regulate acetylation of yeast histone $\mathrm{H} 4$ lysine16 and spreading of heterochromatin. Nat. Genet. 32: 378-383.

Timmers, H.T. and Tora, L. 2005. SAGA unveiled. Trends Biochem. Sci. 30: 7-10.

Trievel, R.C., Rojas, J.R., Sterner, D.E., Venkataramani, R.N., Wang, L., Zhou, J., Allis, C.D., Berger, S.L., and Marmor- stein, R. 1999. Crystal structure and mechanism of histone acetylation of the yeast GCN5 transcriptional coactivator. Proc. Natl. Acad. Sci. 96: 8931-8936.

Zhang, H., Richardson, D.O., Roberts, D.N., Utley, R., Erdjument-Bromage, H., Tempst, P., Cote, J., and Cairns, B.R. 2004. The Yaf9 component of the SWR1 and NuA4 complexes is required for proper gene expression, histone $\mathrm{H} 4$ acetylation, and Htzl replacement near telomeres. Mol. Cell. Biol. 24: 9424-9436. 


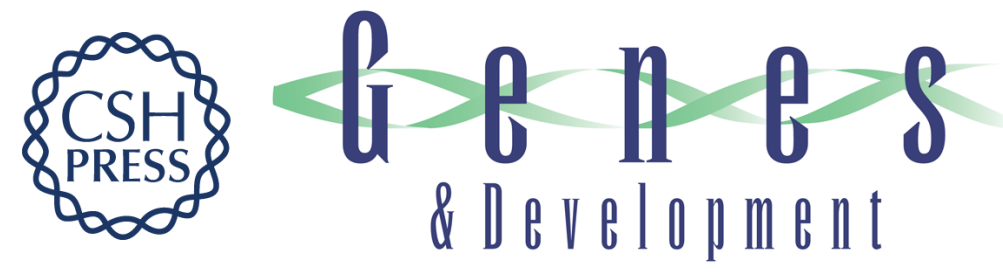

\section{Telomeric heterochromatin boundaries require NuA4-dependent acetylation of histone variant H2A.Z in Saccharomycescerevisiae}

Joshua E. Babiarz, Jeffrey E. Halley and Jasper Rine

Genes Dev. 2006, 20:

Access the most recent version at doi:10.1101/gad.1386306

Supplemental
Material http://genesdev.cshlp.org/content/suppl/2006/03/01/20.6.700.DC1

References This article cites 44 articles, 18 of which can be accessed free at:

http://genesdev.cshlp.org/content/20/6/700.full.html\#ref-list-1

License

Email Alerting Receive free email alerts when new articles cite this article - sign up in the box at the top

Service right corner of the article or click here.

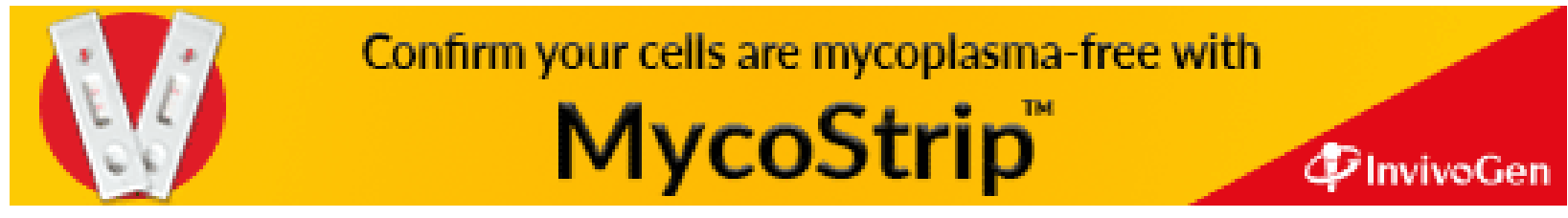

